\title{
Vulnerabilidade social e autocuidado relacionado à prevenção do câncer de mama e de colo uterino
}

\section{Social vulnerability and self-care related to breast and cervical cancer prevention}
Vulnerabilidad social e autocuidado relacionado a la prevención del cáncer de mama y de cuello uterino

\section{Recebido: 10/12/2015 \\ Aprovado: 03/03/2016 \\ Publicado: 01/05/2016}

\author{
Joquebede Cristina Luchetti ${ }^{1}$ \\ Márcia Regina Campos Costa da Fonseca² \\ Maria Cristina Traldi ${ }^{3}$
}

O objetivo do estudo foi analisar a associação entre vulnerabilidade social e o autocuidado através da análise da adesão às ações dos programas de prevenção do câncer de mama e de colo uterino em uma comunidade fechada de mulheres. Estudo transversal com corte no período de 2008 e 2014, com amostra de 495 mulheres $\geq 40$ anos, moradoras da área de abrangência de uma Unidade de Saúde da Família em município do sudeste brasileiro. A média anual de Papanicolaou e mamografias e/ou ultrassonografias foi de 150,0 e 150,6, entre todas pesquisadas respectivamente. Não houve associação significativa entre adesão aos exames preventivos do câncer de mama e de colo uterino e os dois grupos: sem vulnerabilidade social $(77,8 \%)$ e nesta condição $(22,2 \%)$. Conclui-se que as ações da Estratégia de Saúde da Família estão alcançando efetividade em promover a equidade do acesso e facilitar o autocuidado com a saúde dos grupos mais vulneráveis.

Descritores: Vulnerabilidade em saúde; Neoplasias da mama; Neoplasias do colo do útero; Saúde da mulher.

The aim of this study was to analyze the association between social vulnerability and self-care through analyzing the accession to the actions of prevention programs for breast cancer and cervical in a closed community of women. A cross sectional study with cut in the period 2008 and 2014 years and sample of 495 women $\geq 40$ years, living in the coverage area of a Family Health Unit in a city in southeastern Brazil. The average annual Pap smear and mammogram and / or ultrasound were 150.0 and 150.6, respectively. There was no significant association between adherence to preventive exams for breast and cervical cancer and two groups: without social vulnerability (77.8\%) and in this condition (22.2\%). In conclusion, the actions of the Family Health Strategy are reaching effectiveness in promoting equity of access and facilitate self-care for the health of the most vulnerable groups.

Descriptors: Health vulnerability; Breast neoplasms; Uterine cervical neoplasms; Women's health.

El objetivo del estudio fue analizar la asociación entre la vulnerabilidad social y el auto-cuidado mediante el análisis de la adhesión a las acciones de los programas de prevención de cáncer de mama y de cuello de útero en una comunidad cerrada de mujeres. Estudio transversal hecho en el periodo de 2008 al 2014 con muestra de 495 mujeres $\geq 40$ años, que viven en la zona cercana de una Unidad de Salud de la Familia en una ciudad en el sureste del Brasil. El promedio anual del Papanicolaou y mamografía y/o ecografía fue 150,0 y 150,6, respectivamente. No se encontró asociación significativa entre la adhesión a exámenes preventivos para el cáncer de mama y de cuello uterino y dos grupos sin vulnerabilidad social $(77,8 \%)$ y en esta condición (22,2\%). En conclusión, las acciones de la Estrategia Salud de la Familia están alcanzando eficacia en la promoción de la equidad de acceso y facilitar el auto-cuidado de la salud de los grupos más vulnerables.

Descriptores: Vulnerabilidad en salud; Neoplasias de la mama; Neoplasias del cuello uterino , Salud de la mujer.

\footnotetext{
${ }^{1}$ Enfermeira graduada pela Faculdade de Medicina de Jundiaí (FMJ). joquebedeluchetti@gmail.com. Brasil.

2 Enfermeira. Mestre em Farmacologia. Doutora em Ciências Médicas. Professora Adjunta de graduação e do Programa de Mestrado Acadêmico em Ciências da Saúde da Faculdade de Medicina de Jundiaí (FMJ) e Professora da Faculdade São Leopoldo Mandic - Campinas. fonseca100@uol.com.br. Brasil.

3 Enfermeira. Especialista em Medicina Social Preventiva. Mestre e Doutora em Educação. Professora Adjunta da Faculdade de Medicina de Jundiaí (FMJ) e do Programa de Mestrado Acadêmico em Ciências da Saúde. mcristraldi@gmail.com. Brasil.
} 
INTRODUÇÃO

$\mathrm{O}$ câncer é a denominação atribuída a um conjunto de mais de cem doenças que tem em comum o crescimento desordenado de células que invadem tecidos e órgãos. No Brasil, 223.700 mortes por câncer foram contabilizadas no ano de 2014, de um total de 1.318 .000 óbitos, das quais, 104.100 $(7,9 \%)$ ocorreram em mulheres. 0 câncer de mama e o cervico uterino representaram respectivamente $16,8 \%$ e $8,6 \%$ das mortes por câncer em mulheres, ultrapassando $25 \%$ do total de óbitos causados por neoplasias 1 .

Em 10 anos (2003-2013), os óbitos causados pelo câncer de colo do útero cresceram $29,2 \%$ e o de mama, $52 \%$, assim como os decorrentes da doença localizados em outros sítios. 0 crescimento reflete o aumento da incidência na população, além de outros fatores relacionados à demora no diagnóstico ou início do tratamento oportuno ${ }^{2,3}$.

O câncer de colo de útero apresenta um dos mais altos potenciais de cura, chegando a $100 \%$, quando diagnosticado e tratado em estádios iniciais ou em fases precursoras; sua incidência aumenta a partir dos 30 anos de idade ${ }^{4}$. 0 câncer de mama, se diagnosticado precocemente, também apresenta prognóstico favorável e elevado percentual de cura ${ }^{5}$.

São os dois tipos de câncer com políticas preventivas bem delineadas, medidas de rastreamento sistematizadas e amplamente divulgadas em campanhas de conscientização entre a população $0^{6,7}$.

As ações de rastreamento do câncer de colo uterino são destinadas a mulheres que iniciaram a atividade sexual, principalmente aquelas na faixa etária de 25 a 64 anos. São preconizados dois exames em anos consecutivos e, em caso de resultados negativos seguidos, o intervalo entre os exames passa para três anos, enquanto permanecer a condição de negatividade ${ }^{7}$. A adesão, entretanto, atinge mulheres mais jovens; em 2010, aproximadamente 18\% dos exames de Papanicolaou foram realizados em mulheres com menos de 25 anos $^{8,9}$.
Atualmente as medidas de rastreamento do câncer de mama são baseadas unicamente na mamografia, considerado exame padrão-ouro em relação a outros métodos de imagem, devido ao seu potencial de detectar a maioria dos tipos de câncer, antes mesmo que seja perceptível alguma nodulação à palpação. É recomendada a mulheres de $50 \mathrm{a}$ 69 anos a cada dois anos, exceto para as que pertencem a um dos grupos de risco tais como: histórico familiar da doença, menarca precoce, primeira gravidez após os 30 anos, menopausa tardia (após os 50 anos de idade), e nuliparidade; nesses casos recomenda-se que a mamografia passe a ser realizada a partir dos 35 anos $^{10}$.

Entre os meses de janeiro e outubro de 2013, 4,1 milhões de exames mamográficos, somados os de rastreamento e de diagnóstico, foram realizados por mulheres em todas as faixas etárias no Brasil e os registros indicaram um aumento de $25 \%$ no número de mamografias realizadas pelo SUS entre os anos de 2010 e $2012^{11}$.

O nível de regulamentação das medidas de prevenção de câncer de mama e de colo uterino permitiu criar no país um sistema de monitoramento das ações desenvolvidas. O Sistema de Informação do Câncer do Colo do Útero - SISColo e o Sistema de Informação do Controle do Câncer de Mama - SISMama são sistemas informatizados que reúnem informações sobre os programas de prevenção dos dois tipos de câncer que mais afetam a população feminina, embora ainda careçam de registros que retratem a totalidade das ações realizadas pelos serviços, especialmente pelo setor privado de saúde ${ }^{12,13}$.

A existência desses dois sistemas informatizados, a característica da regulamentação bem delineada das políticas públicas oncológicas, assim como a disponibilidade de acesso das ações sobre os dois programas no âmbito da atenção básica constituíram os principais motivos que justificaram a adoção dos mesmos para analisar o autocuidado com a saúde da mulher, influenciadores na realização desta pesquisa. 
Muitos estudos realizados no Brasil têm se dedicado a avaliação da adesão às medidas de prevenção e controle do câncer de mama e de colo uterino, mas o foco deles recai especialmente sobre um ou outros programas de rastreamento, deixando uma lacuna no conhecimento dos principais aspectos que envolvem a adesão, particularmente os fatores sociodemográficos para além das características pessoais, de renda e escolaridade, que também podem influenciar o acesso às ações oferecidas no âmbito da atenção básica ${ }^{4,5,14}$.

Partindo dessa lacuna do conhecimento, uma pergunta norteou o desenvolvimento deste estudo: "mulheres que praticam ações de autocuidado relativas à prevenção do câncer de mama e de colo do útero constituem o segmento da população feminina socialmente menos vulnerável?". Em outras palavras, mulheres que convivem em núcleos familiares sob maior risco de adoecimento devido a condição especial de comprometimento da saúde de um ou mais de seus membros são as que menos dedicam tempo à própria saúde?

A ideia de estudar o autocuidado associado à vulnerabilidade social surgiu a partir do conceito desta última expressão que atribui aos que pertencem a esse segmento menor poder de enfrentamento de uma situação de risco e de tomada de decisão.

A vulnerabilidade social está comumente relacionada aos processos de exclusão, discriminação, enfraquecimento dos grupos sociais e de sua capacidade de reação, tornando estreita a relação entre vulnerabilidade social e risco ${ }^{15}$.

Para responder ao questionamento proposto, este estudo recuperou os registros de mamografia e/ou ultrassonografia e do exame de Papanicolaou, de uma dada população feminina para verificar o padrão de adesão às medidas de prevenção do câncer de colo de útero e de mama, buscando caracterizar a atitude de autocuidado dessas mulheres em dois grupos distintos: o de mulheres oriundas de famílias em situação de vulnerabilidade social e daquelas sem esta condição.

Para tanto, o objetivo principal deste estudo foi o de analisar a associação entre vulnerabilidade social e adesão aos programas de prevenção do câncer de mama e de colo uterino em mulheres de uma comunidade, no período de sete anos (de 2008 a 2014).

Trabalhou-se com a hipótese de que as mulheres socialmente não vulneráveis são também as que mais praticam o autocuidado com a saúde e realizam com regularidade a mamografia e/ou a ultrassonografia e o exame de Papanicolaou para rastreamento dos cânceres de mama e de colo uterino.

\section{MÉTODO}

Trata-se de um estudo transversal, realizado com mulheres residentes na área de abrangência e usuárias em uma Unidade de Saúde da Família (USF) de um município do interior paulista, no sudoeste brasileiro, no período de sete anos, entre 2008 e 2014.

A amostra foi definida a partir de lista de 657 mulheres com idade igual ou superior a 40 anos, moradoras das seis microáreas que compõem a região de abrangência da Unidade de Saúde da Família estudada. Trata-se de um cadastro utilizado pelos Agentes Comunitários de Saúde para agendamento anual dos exames mamográficos de rastreamento do câncer de mama e a busca ativa das faltosas. Cabe destacar que o município orienta as unidades de atenção básica realizar o rastreamento do câncer de mama a partir dos 40 anos.

Foram excluídas 162 mulheres por não frequentarem a Unidade, resultando numa amostra de 495 mulheres, representando 75,3\% das cadastradas.

Aspecto que limita a generalização dos resultados deste estudo está na opção da amostra ser composta por mulheres com idade $\geq 40$ anos, adotada pela oportunidade da existência do cadastro, não distinguindo a população alvo do exame Papanicolaou; a diferença de faixa etária entre os públicos alvo foi considerada baixa como fator de confundimento, uma vez que o estudo buscou 
observar a prática do autocuidado a partir da frequência de realização de exames preconizados nas políticas públicas de saúde e não $o$ de estabelecer a prevalência dos mesmos.

As fontes de coleta foram o Sistema Informatizado de Informações de Saúde do Município (SIIM), os prontuários das usuárias, o Sistema de Informação do Câncer de Mama (SISMama) e Sistema de Informação do Câncer do Colo do Útero (SISColo), o livro de registro da Unidade e os relatórios produzidos pelos Agentes Comunitários de Saúde.

Para orientar a coleta de dados foi elaborado um roteiro estruturado, incluindo as variáveis sociodemográficas de interesse para o estudo: idade, escolaridade, estado civil, renda, profissão, número de filhos e risco familiar (Escala de Coelho-Savassi); as variáveis relativas ao autocuidado foram: data dos exames realizados de mamografia e/ou de ultrassonografia e da coleta de material para exame de Papanicolaou.

0 parâmetro para se atribuir a condição de vulnerabilidade social foi a Escala de Coelho-Savassi (ERF-CS), adotada pelo Ministério da Saúde para classificação do risco social na Estratégia de Saúde da Família. A Escala de Risco Familiar de Coelho-Savassi (ERF-CS) é um instrumento de estratificação da vulnerabilidade familiar, desenvolvido em Contagem, Minas Gerais, elaborado a partir da ficha A do Sistema de Informação da Atenção Básica (SIAB). A escala é preenchida pelo Agente Comunitário de Saúde no cadastramento das famílias para determinar seu risco social e de saúde e reflete o potencial de adoecimento de cada núcleo familiar. A Escala de Coelho atribui risco familiar pontuando as famílias que possuem entre seus membros: acamados, deficiência física, deficiência mental, baixas condições de higiene, desnutrição, vícios (Drogadição/ Tabagismo/Etilismo), desemprego, analfabetismo, criança menor que 1 ano, idosos maiores de 70 anos, hipertensos e diabéticos ${ }^{16}$.

A coleta de dados foi realizada entre os meses de março e abril de 2015 e os dados foram digitados em planilha Excel e para o tratamento estatístico foi usado o programa SAS (Statistical Analysis System) versão 9.2.

A análise estatística foi feita utilizandose frequências absolutas (n) e relativas (\%), medidas de tendência central (média e mediana) e de dispersão (desvio-padrão) para as variáveis quantitativas. Para avaliar o grau de dependência entre os grupos com e sem risco social e as variáveis de interesse do estudo foi utilizado o teste Qui-quadrado e o Odds Ratio, utilizando como controle as mulheres sem risco social. 0 nível de significância assumido nas análises estatísticas foi de $5 \%$.

0 protocolo de pesquisa foi aprovado pelo Comitê de Ética sob o $\mathrm{n}^{0}$. 42499215.8.0000.5412, e a pesquisa seguiu as recomendações da Resolução CNS no 466/12 e da Declaração de Helsinki.

\section{RESULTADOS}

A média etária das 495 participantes foi $55,8 \pm 10,89$ anos (40-90); $34,9 \%$ na faixa de 40 e 49 anos, $31,2 \%$ entre 50 e 59 anos, $21,9 \%$ entre 60 e 69 anos e 12,0\%, com idade igual ou superior a 70 anos. Não foi identificada a idade de duas participantes. A expressiva maioria é caucasiana $(92,7 \%)$ e 3,5\% afrodescendente; as demais $(3,9 \%)$ são de origem indígena ou asiática. Para seis mulheres não foi possível identificar a etnia/cor da pele.

Do total, $70,3 \%$ viviam com seus companheiros, seja por meio de contrato de casamento ou em situação de união estável; 9,4\% eram solteiras, 8,6\% separadas e 9,0\% viúvas. Para sete mulheres o estado civil não foi identificado.

A maioria das mulheres $(62,4 \%)$ não tinha atividade no mercado de trabalho, 31,7\% tinham uma profissão definida e 5,9\% eram aposentadas. A análise da escolaridade indicou que $80,8 \%$ cursaram o Ensino Fundamental, tendo concluído ou não esse grau de ensino, o mesmo ocorreu com 8,4\% que cursou o Ensino Médio e 2,5\% com Ensino Superior; 8,4\% eram analfabetas. Não foram encontrados registros da escolaridade de seis mulheres. 
Na Tabela 1, é possível verificar que de 2008 a 2014 houve uma crescente inclusão de mulheres nos programas de prevenção dos cânceres de colo uterino e de mama. Até o ano de 2010 o percentual de mulheres atendidas para a realização do exame de citologia oncótica do colo do útero atingiu pouco mais de $30,0 \%$ do da população estudada. A partir de 2011 nota-se um aumento no percentual de participantes, atingindo o máximo de inclusão no ano de 2012, com 44,0\% de mulheres atendidas.
0 percentual de mulheres atendidas para rastreamento do câncer de mama foi maior que no de câncer de colo uterino em quatro dos sete anos estudados, tendo tingido $50,1 \%$ e $44,0 \%$, respectivamente em 2012, ano de melhor desempenho dos programas. A média anual de exames realizados pelas 495 mulheres foi semelhante, 150,0 citologias oncóticas do colo do útero e de 150,6 mamografias (MMG) e/ou ultrassonografia (USG) (Tabela 1).

Tabela 1. Exames de Papanicolaou e de MMG e/ou USG, segundo ano de realização, em mulheres com 40 anos ou mais, usuárias de uma USF em um município do interior Paulista, 2008-2014.

\begin{tabular}{lrcrc}
\multirow{2}{*}{ ANO } & \multicolumn{2}{c}{ Papanicolaou } & \multicolumn{2}{c}{ MMG/USG } \\
& $\mathrm{n}$ & $\%$ & $\mathrm{n}$ & $\%$ \\
\hline 2008 & 102 & 20,6 & 4 & 0,8 \\
2009 & 88 & 17,8 & 114 & 23,0 \\
2010 & 122 & 24,6 & 143 & 28,9 \\
2011 & 178 & 36,0 & 214 & 43,2 \\
2012 & 218 & 44,0 & 248 & 50,1 \\
2013 & 171 & 34,5 & 166 & 33,5 \\
2014 & 171 & 34,5 & 165 & 33,3 \\
Total & 1050 & \multicolumn{3}{c}{1054} \\
\hline
\end{tabular}

Entre as participantes, considerado o risco social pela escala de Coelho-Savassi, 385 (77,8\%) mulheres pertenciam a famílias sem risco social e $110(22,2 \%)$ com algum grau de risco atribuído. Das famílias com risco social, $57(51,8 \%)$ tinham risco $1 ; 33(30,0 \%)$ risco 2 ; e $20(18,2 \%)$ risco 3 (Tabela 2 ).

A microárea 4 é a que concentra maior população de mulheres da amostra, mas a que possui maior percentual de famílias em risco para a saúde é a 5 , com $30,2 \%$ do total de famílias apresentando algum tipo de fator social que a inclui entre as vulneráveis. Contudo, residir em uma ou outra microárea não apresentou diferença significativa para risco social $(p=0,8634)$, assim como também não foi significativo $(\mathrm{p}=0,9666)$ ter grau maior ou menor de risco (R1, R2, R3) e residir nas diferentes microáreas, apesar de algumas apresentarem concentração maior de famílias/mulheres em risco social, como mostra a Tabela 2.

A média etária das mulheres nos grupos foi semelhante, $55,9 \pm 10,64(40-88)$ para as sem risco social e de 55,8 $\pm 11,76$ (41-90) para as com risco social.

0 percentual de mulheres que realizaram MMG/USG e o Papanicolaou foi maior entre as pertencentes às famílias sem risco social em todos os anos estudados e isto já era esperado porque elas constituem a expressiva maioria $(77,8 \%)$. 
Tabela 2. Mulheres segundo a microárea (MA) de residência e o risco social, de uma USF em um município do interior Paulista, 2014.

\begin{tabular}{lrlllrlrll}
\hline Micro & \multicolumn{2}{l}{ Mulheres } & \multicolumn{2}{l}{ Sem Risco } & \multicolumn{2}{c}{ Com Risco } \\
\cline { 2 - 10 } Áreas & \multicolumn{1}{c}{ N } & $\%$ & R 0 & $\%$ & \multicolumn{1}{c}{ n } & $\%$ & R 1 & R 2 & R 3 \\
\hline MA 1 & 56 & 11,3 & 49 & 87,5 & 7 & 12,5 & 3 & 3 & 1 \\
MA 2 & 92 & 18,6 & 73 & 79,3 & 19 & 20,7 & 10 & 7 & 2 \\
MA 3 & 58 & 11,7 & 49 & 84,5 & 9 & 15,5 & 5 & 1 & 3 \\
MA 4 & 115 & 23,2 & 86 & 74,8 & 29 & 25,2 & 13 & 8 & 8 \\
MA 5 & 86 & 17,4 & 60 & 69,8 & 26 & 30,2 & 13 & 9 & 4 \\
MA 6 & 88 & 17,8 & 68 & 79,1 & 20 & 22,7 & 13 & 5 & 2 \\
\hline
\end{tabular}

Os resultados apresentados nas Tabelas 3 e 4 indicam não haver diferença significativa na adesão aos dois programas, considerando o risco social como desfecho e, mostrando que a adesão das mulheres aos programas de prevenção de câncer de mama e de colo uterino não difere, quando analisada pela perspectiva da condição de vulnerabilidade social. Sugerem que o fato de terem pessoas com algum tipo de deficiência ou dependência em conviver com familiar desempregado, por mais que comprometa a rotina e demande maior dedicação da mulher, não interfere na agenda anual de autocuidado, ao menos no que se refere aos exames de Papanicolaou e MMG.

\section{DISCUSSÃO}

A vulnerabilidade social pode ser compreendida como o reflexo das condições de bem-estar social e mantém relação direta com aspectos sociopolíticos e culturais de forma combinada, incluído o acesso a informações, o nível de escolaridade, a disponibilidade de recursos materiais e o poder de enfrentamento das barreiras culturais, entre outros fatores. Esse conjunto de aspectos combinados intensifica a relação entre vulnerabilidade e risco $^{14}$.
0 risco é usado pelos epidemiologistas em associações a grupos ou populações ${ }^{17}$. Pode ser imediato ou interferir na perda da qualidade de vida pela ausência de ações preventivas, resultando no risco social, ou seja, na capacidade dos indivíduos de assegurar por si mesmos sua independência social ${ }^{18,19}$.

Aplicado às mulheres, a vulnerabilidade social atribuída pela Escala de Coelho-Savassi pode culminar em maior dificuldade na definição de espaço na agenda diária para buscar realizar ações voltadas para si, como o cuidado com a saúde, o que poderia aumentar o risco às doenças como o câncer de mama e de colo uterino pela demora na deteç̧ão dos mesmos $^{5}$.

0 autocuidado é definido como a prática de cuidados executados pela pessoa com o objetivo de manter a saúde e o bem-estar. É a realização de ações direcionadas a si mesmo buscando fortalecer os aspectos de uma vida saudável ${ }^{20}$.

$\mathrm{Na}$ saúde, as necessidades de autocuidado surgem tanto do estado patológico, ou pela necessidade de procedimentos para o diagnóstico $\mathrm{e}$ tratamento da patologia 21 . 
Tabela 3. Exames de Papanicolaou, segundo ano e risco social, em mulheres com 40 anos ou mais, usuárias da Unidade de Saúde da Família, em município do interior Paulista, 2008-2014.

\begin{tabular}{llrrrlll}
\hline Ano & \multicolumn{6}{c}{ Exame de Papanicolaou } & \\
\hline 2008 & & SIM & NÃO & Total & OR* & IC 95\% & Valor P \\
& C/ risco & 23 & 87 & 110 & 1,024 & $(0,6076-1,7257)$ & $\mathrm{p}=0,9645$ \\
& S/ risco & 79 & 306 & 385 & & & \\
& Total & 102 & 393 & 495 & & & \\
2009 & & & & & & & \\
& C/ risco & 19 & 91 & 110 & 0,9562 & $(0,5469-1,6720)$ & $\mathrm{p}=0,9875$ \\
& S/ risco & 69 & 316 & 385 & & & \\
& Total & 88 & 407 & 495 & & & \\
2010 & & & & & & & \\
& C/ risco & 27 & 83 & 110 & 0,993 & $(0,6070-1,6245)$ & $\mathrm{p}=0,9223$ \\
& S/ risco & 95 & 290 & 385 & & & \\
& Total & 122 & 373 & 495 & & & \\
2011 & & & & & & & \\
& C/ risco & 38 & 72 & 110 & 0,9236 & $(0,5922-1,4405)$ & $\mathrm{p}=0,8120$ \\
& S/ risco & 140 & 245 & 385 & & & \\
& Total & 178 & 317 & 495 & & & \\
2012 & & & & & & & \\
& C/ risco & 52 & 58 & 110 & 1,1828 & $(0,7731-1,8096)$ & $\mathrm{p}=0,5058$ \\
& S/ risco & 166 & 219 & 385 & & & \\
& Total & 218 & 277 & 495 & & & \\
2013 & & & & & & & \\
& C/ risco & 37 & 73 & 110 & 0,9094 & $(0,6068-1,4855)$ & $\mathrm{p}=0,9095$ \\
& S/ risco & 134 & 251 & 385 & & & \\
& Total & 171 & 324 & 495 & & & \\
2014 & & & & & & & \\
& C/ risco & 40 & 70 & 110 & 1,108 & $(0,7121-1,7238)$ & $\mathrm{p}=0,7331$ \\
& S/ risco & 131 & 254 & 385 & & & \\
& Total & 171 & 324 & 495 & & & \\
\hline
\end{tabular}

*Controle sem risco 
Tabela 4. Exames de MMG e/ou USG, segundo o ano e o risco social, em mulheres com 40 anos ou mais, usuárias de uma USF, em um município do interior Paulista, 2008-2014.

\begin{tabular}{llrrllll}
\hline Ano & & \multicolumn{6}{c}{ MMG e/ou USG } \\
\hline 2008 & & SIM & NÃO & Total & OR* & IC 95\% & Valor P \\
& C/ risco & 0 & 110 & 110 & & & \\
& S/ risco & 4 & 381 & 385 & & & \\
& Total & 4 & 491 & 495 & & & \\
2009 & & & & & & & \\
& C/ risco & 24 & 91 & 115 & 0,8498 & $(0,5113-1,4126)$ & $\mathrm{p}=0,6158$ \\
& S/ risco & 90 & 290 & 380 & & & \\
& Total & 114 & 381 & 495 & & & \\
2010 & & & & & & & \\
& C/ risco & 28 & 68 & 96 & 1,1137 & $(0,6797-1,8248)$ & $\mathrm{p}=0,7642$ \\
& S/ risco & 105 & 284 & 389 & & & \\
& Total & 133 & 352 & 485 & & & \\
2011 & & & & & & & \\
& C/ risco & 35 & 67 & 102 & 0,6245 & $(0,3965-0,9838)$ & $\mathrm{p}=0,0538$ \\
& S/ risco & 179 & 214 & 393 & & & \\
& Total & 214 & 281 & 495 & & & \\
2012 & & & & & & & \\
& C/ risco & 51 & 57 & 108 & 0,8629 & $(0,5630-1,3228)$ & $\mathrm{p}=0,5701$ \\
& S/ risco & 197 & 190 & 387 & & & \\
& Total & 248 & 247 & 495 & & & \\
2013 & & & & & & & \\
& C/ risco & 33 & 80 & 113 & 0,7723 & $(0,4890-1,2195)$ & $\mathrm{p}=0,3188$ \\
& S/ risco & 133 & 249 & 382 & & & \\
& Total & 166 & 329 & 495 & & & \\
2014 & & & & & & & \\
& C/ risco & 33 & 72 & 105 & 0,8958 & $(0,5642-1,4224)$ & $\mathrm{p}=0,7265$ \\
& S/ risco & 132 & 258 & 390 & & & \\
& Total & 165 & 330 & 495 & & & \\
\hline
\end{tabular}

*Controle sem risco

Os dois grupos de mulheres estudadas: as que pertencem às famílias sem vulnerabilidade social $(77,8 \%)$ e as que vivem com algum grau de risco social/vulnerabilidade social $(22,2 \%)$ tiveram acesso equânime para realizar os exames de rastreamento do câncer de mama e de colo uterino, pois não foi verificada qualquer associação significativa entre as variáveis risco social e MMG/USG ou Papanicolaou, o que sugere que os dois grupos aderem de maneira semelhante às medidas preventivas, contrariando as proposições sobre aumento de risco e vulnerabilidade social $18,19,22$.

Por outro lado, a contradição que os resultados inicialmente sugerem remete a uma questão bastante cara ao campo da Saúde Coletiva que propõe ações que partem da constatação de condições específicas de vulnerabilidade para construir práticas qualificadas, instrumentos e tecnologias que possam suprir as necessidades de determinados grupos sociais ${ }^{23}$.

A proposta de utilizar a Escala de Coelho-Savassi na Estratégia de Saúde da 
Família tem o claro propósito de identificar as famílias em situação de vulnerabilidade e por meio de ações intencionais e planejadas em equipe, intervir estrategicamente para reduzir a desigualdade no acesso aos serviços e aumentar o poder de enfrentamento das situações cotidianas, reduzindo a exclusão, a discriminação através do acolhimento e da corresponsabilidade com a saúde da população ${ }^{5}$.

Neste estudo, a adesão das mulheres dos dois grupos às medidas de rastreamento do câncer de mama e de colo uterino pode ser indicativa da efetividade da equipe da ESF local em atuar na perspectiva do oferecimento equânime das ações de saúde, investindo mais esforços junto ao segmento que mais necessita - o de maior vulnerabilidade social.

Estudo de base populacional no Brasil mostrou que a mamografia foi realizada por $60 \%$ das mulheres com idade entre 50 e 69 anos e o exame Papanicolaou, por $84,5 \%$ da população alvo e apontou como fatores dificultadores do acesso à mamografia a distância geográfica dos serviços ofertados, a estrutura familiar, caracterizada pela condição econômica e falta de estímulos para o cuidado da saúde, além da percepção sobre a relevância do cuidado 22,24 .

$\mathrm{Na}$ população feminina estudada, o exame de Papanicolaou é realizado na USF, próxima da residência e no momento da consulta, enquanto que a mamografia depende de um agendamento em um centro de especialidades, exigindo o deslocamento das mulheres do bairro, o que provavelmente dificulta o acesso ao exame, especialmente para aquelas provenientes de famílias em situação de risco social.

No entanto, os resultados de nosso estudo contrariam outros que afirmam ser maior adesão à MMG/USG que ao Papanicolaou, pois a média anual dos dois exames realizados nos período de sete anos de acompanhamento foi semelhante, com variação de seis décimos.

As equipes da ESF são fundamentais para a adesão da família ao autocuidado, principalmente nos grupos vulneráveis, que mais demandam orientações e cuidados e devem estar preparadas para agir observando as particularidades de cada situação, buscando adequar-se às necessidades evidenciadas, principalmente aquelas relacionadas à baixa escolaridade que dificulta a compreensão das informações. O nível de escolaridade pode influenciar na realização de medidas de prevenção do câncer de mama e, por consequência, no atraso para a identificação do tumor $25-27$.

Neste estudo, a maioria das mulheres $(80,8 \%)$ não chegou a cursar o ensino médio, ou seja, constitui uma população com baixa escolaridade.

Nos Estados Unidos, mulheres que nunca fizeram mamografia apresentam menor escolaridade e maior vulnerabilidade econômica $^{26}$. No Brasil, 36,8\% das mulheres sem escolaridade e com mais de 24 anos haviam realizado o exame clínico das mamas contra $90,0 \%$ das mulheres com 15 anos ou mais de escolaridade na mesma faixa etária ${ }^{28}$.

Nesse contexto, destaca-se mais uma vez a o papel da ESF de intervir em situações específicas, como nas abordagens educativas dinâmicas e lúdicas que visam a atingir grupos específicos de mulheres com baixa escolaridade buscando melhorar o seu conhecimento e desenvolver atitudes e práticas que promovam o autocuidado com a saúde.

Somando-se às vulnerabilidades relativas à baixa escolaridade, os resultados mostraram que a maioria das mulheres $(62,4 \%)$ não tem atividade no mercado de trabalho, portanto, não possui fonte própria de renda, sendo dependente financeiramente de seus companheiros, visto que, 70,3\% delas são casadas ou vivem em situação de união estável, expondo toda à coorte de mulheres a uma situação de vulnerabilidade social, independente daquela atribuída pela Escala de Coelho, conforme mostram estudos que associaram menor cobertura de Papanicolaou e mamografia em mulheres com baixa condição econômica e a baixa escolaridade 29,30 . 
Os resultados deste estudo não confirmaram a hipótese de que o autocuidado com a saúde é maior entre mulheres sem risco social, visto que não houve diferença estatisticamente significativa entre apresentar ou não vulnerabilidade social. Contudo, corrobora os estudos que associam a vulnerabilidade social configurada pelo baixo poder aquisitivo e de escolaridade a menores coberturas dos programas de prevenção do câncer de mama e colo de útero, pois esta não ultrapassa os $50,0 \%$ entre as mulheres da coorte, independentemente de integrarem ou não o grupo de risco social de Coelho-Savassi.

Ressalta-se que a Organização Mundial da Saúde (OMS) e o Ministério da Saúde estabelecem em 70,0\% a meta de mulheres com 50 a 69 anos realizem uma mamografia a cada dois anos e em 80,0\% de mulheres entre 25 a 64 anos, o exame de Papanicolaou a cada três anos ${ }^{24,31}$. O número de mulheres que realiza os exames anualmente vem aumentando na unidade de saúde estudada, mas ainda se encontra muito distante do considerado ideal, que é a totalidade das mulheres da área.

A adesão às medidas preventivas instituídas nos programas de câncer de colo uterino e de mama apresentou evolução positiva ao longo dos sete anos de acompanhamento e ambos os programas apresentaram médias anuais semelhantes. Não se encontrou diferença estatisticamente significativa entre a associação de autocuidado e vulnerabilidade social, atribuído pela Escala de Coelho-Savassi, entre as mulheres da comunidade estudada.

As ações desenvolvidas pela Estratégia de Saúde da Família estão sendo eficazes no propósito de promover a equidade do acesso das mulheres e facilitar o autocuidado com a saúde dos grupos mais vulneráveis, ainda que a adesão aos mesmos se mostrem baixos, não ultrapassando $50 \%$ das mulheres com idade $\geq$ 40 anos.

\section{CONCLUSÃO}

A vulnerabilidade social atribuída pelo risco social da escala de Coelho-Savassi não se constituiu em justificativa para a adoção dos programas de rastreamento dos cânceres de mama e colo uterino.

As estratégias adotadas pela equipe de saúde local devem ser intensificadas para ampliar o acesso das medidas de rastreamento dos cânceres de colo uterino e de mama ao conjunto de mulheres da área de abrangência.

\section{REFERÊNCIAS}

1. World Health Organization (WHO). Cancer Country Profiles, 2014 [Internet]. Geneva: WHO; 2016. [Internet] [Acesso em 2015 Nov 04]. Disponível em: www.who.int/cancer/countryprofiles/en/

2. Ministério da Saúde (Br). Instituto Nacional de Câncer José de Alencar Gomes da Silva. ABC do Câncer: abordagens básicas para o controle do câncer. [Internet]. Rio de Janeiro: INCA; 2011 [citado em 03 nov 2015]. Disponível em: http://bvsms.saude.gov.br/bvs/publicacoes/abc_d o_cancer.pdf.

3. Ministério da Saúde (Br). Instituto Nacional de Câncer José de Alencar Gomes da Silva (INCA). Atlas online da mortalidade, 2014. [Internet]. Rio de Janeiro: INCA; 2014 [citado em 10 out de 2015] Disponível

em: <https://mortalidade.inca.gov.br/MortalidadeWeb /pages/Modelo01/consultar.xhtml\#panelResultad o>

4. Ministério da Saúde (Br). Instituto Nacional de Câncer. Inquérito domiciliar sobre comportamentos de risco e morbidade referida de doenças e agravos não transmissíveis. Brasil, 15 capitais e Distrito Federal 2002-2003 [Internet]. Rio de Janeiro: INCA; 2005 [citado em 22 ago 2015]. Detecção precoce de câncer de colo do útero e mama; p.121-131. Disponível em: http://www.inca.gov.br/inquerito/docs/detec_ma ma_colo.pdf.

5. Ministério da Saúde (Br). Secretaria de Atenção à Saúde. Departamento de Atenção Básica. Controle dos cânceres do colo do útero e da mama [Internet]. 2ed. Brasília (DF): Departamento de Atenção Básica; 2013 [citado em 12 ago 2015]. (Cadernos de Atenção Básica; n.13). Disponível em: http://bvsms.saude.gov.br/bvs/publicacoes/contr ole_canceres_colo_utero_2013.pdf. 
6. Ministério da Saúde (Br). Portaria no $1228 / 30$ out 2012. Regulamenta a habilitação para o Programa de Mamografia Móvel, instituído pela Portaria $\mathrm{n}^{\circ}$ 2.304/GM/MS, de 4 de outubro de 2012 [Internet]. Brasília (DF): Ministério da Saúde; 2012 [citado em 21 ago 2015]. Disponível em: http://bvsms.saude.gov.br/bvs/saudelegis/sas/20 12/prt1228_30_10_2012.html.

7. Ministério da Saúde (Br). Secretaria de Atenção a Saúde. Departamento de Atenção Básica. Controle dos cânceres do colo do útero e da mama. Brasília (DF): Ministério da Saúde; 2006. 20p. (Cadernos de Atenção Básica; 13).

8. Martins LFL, Thuler LCS, Valente JG. Cobertura do exame de papanicolaou no Brasil e seus fatores determinantes: uma revisão sistemática da literatura. Rev Bras Ginecol Obstet. 2005; 27(8):485-92.

9. Ministério da Saúde (Br). Controle dos cânceres do colo do útero e da mama. 2ed. Brasília (DF): Ministério da Saúde; 2012. (Cadernos de Atenção Básica; 13).

10. Ministério da Saúde (Br). Instituto Nacional de Câncer. Controle do câncer de mama: documento de consenso [Internet]. Rio de Janeiro: INCA; 2004 [citado em 13 ago 2015]. Disponível em:

http://www2.inca.gov.br/wps/wcm/connect/tipo sdecancer/site/home/mama+/prevencao.

11. Portal Brasil (Brasil). SUS assegura às mulheres exames de mamografia [Internet]. Brasília (DF): Portal Brasil; 2014 [citado em 10 out 2015]. Disponível em: http://www.brasil.gov.br/saude/2014/02/susassegura-as-mulheres-exames-de-mamografia. 12. Ministério da Saúde (Br). Portaria n. 408, de 30 de agosto de 1999. Programa Nacional de Prevenção ao Câncer de Colo Uterino. Diário Oficial da União, Brasília (DF), 2 ago1999. Seção 1. p.14. 13. Ministério da Saúde (Br). Portaria no 779/31 dez 2008. Institui o Sistema de Informação do Câncer de Mama (SISMAMA) [Internet]. Brasília (DF): Ministério da Saúde; 2008 [citado em 12 out 2015]. Disponível em: http://bvsms.saude.gov.br/bvs/saudelegis/sas/20 08/prt0779_31_12_2008.html

14. Lima-Costa MF, Matos DL. Prevalência e fatores associados à realização da mamografia na faixa etária de 50-69 anos: um estudo baseado na Pesquisa Nacional por Amostra de Domicílios (2003). Cad Saúde Pública. 2007; 23(7):1665-73.

15. Palma A, Mattos UAO. Contribuições da ciência pós normal à saúde pública e a questão da vulnerabilidade social. Hist Ciênc SaúdeManguinhos [Internet]. 2001 [citado em 09 out 2015]; 8(3):567-590. Disponível em: http://www.scielo.br/pdf/hcsm/v8n3/7645.pdf. 16. Coelho FLG, Savassi LCM. Aplicação de Escala de Risco Familiar como instrumento de priorização de visitas domiciliares. Rev Bras Med Fam Comunidade. 2004; (2):24-8.

17. Yunes MAM, Szymanski H. Resiliência: noção, conceitos afins e considerações críticas. In: Tavares J. (Org.). Resiliência e educação. 2 ed. São Paulo: Cortez; 2001.

18. Castel R. A insegurança social: o que é ser protegido? Petrópolis: Vozes; 2005.

19. Janczura R. Risco ou vulnerabilidade social? Textos Contextos (Porto Alegre) [Internet], 2012 [citado em 12 ago 2015]; 11(2):301-308. Disponível em: http://revistaseletronicas.pucrs.br/ojs/index.php/ fass/article/view/12173/8639.

20. Bub MBC, Medrano C, Silva CD, Wink S, Liss PE, Santos EKA. A noção de cuidado de si mesmo e o conceito de autocuidado na enfermagem. Texto \& Contexto Enferm. [Internet]. 2006 [citado em 10 out 2015]; 15(Esp):152-7. Disponível em: http://www.scielo.br/pdf/tce/v15nspe/v15nspea 18.

21. Orem D. Nursing: concepts of practice. 6 ed. St. Louis: Mosby; 2001. 22. Oliveira EXG, Pinheiro RS, Melo ECP, Carvalho MS. Condicionantes socioeconômicos e geográficos do acesso à mamografia no Brasil, 20032008. Ciênc Saúde Coletiva. 2011; 16(9):3649-64.

23. Bertolozzi MR, Nichiata LYI, Takahashi RF, Ciosak SI, Hino P, Val LF et al. Os conceitos de vulnerabilidade e adesão na Saúde Coletiva. Rev Esc Enferm USP [Internet]. 2009 [citado em 03 out 2015]; 43(Esp2):1326-30. Disponível em: http://www.scielo.br/pdf/reeusp/v43nspe2/a31v 43s2.pdf.

24. Instituto Brasileiro de Geografia e Estatística (IBGE). Pesquisa Nacional por Amostra de Domicílios (PNAD), 2008 - Suplemento Saúde [Internet]. Rio de Janeiro: IBGE; 2011 [citado em 10 out 2015]. Disponível em: http://www.ibge.gov.br/home/estatistica/populac ao/panorama_saude_brasil_2003

_2008/default.shtm.

25. Pinheiro AB, Lauter DS, Medeiros GC, Cardozo IR, Menezes LM, Souza RMB, et al. Câncer de mama em mulheres jovens: análise de 12.689 Casos. Rev Bras Cancerol. 2013; 59(3):351-9. 
26. Schootman M, Jeffe DB, Reschke AH, Aft RL. Disparities related to socioeconomic status and access to medical care remain in the United States among women who never had a mammogram. Cancer Causes Control. 2003; 14(5):419-25.

27. Höfelmann DA, Anjos JC. Autoavaliação de Saúde e câncer de mama em mulheres de cidade do Sul do Brasil. Rev Bras Cancerol. 2012; 58(2):20922.

28. Ministério da Saúde (Br). Portaria no 874, de 16 de Maio de 2013. Institui a Política Nacional para a Prevenção e Controle do Câncer na Rede de Atenção à Saúde das Pessoas com Doenças Crônicas no âmbito do Sistema Único de Saúde (SUS) [internet]. Brasília (DF): Ministério da Saúde; 2013 [citado em 22 ago 2015]. Disponivel em: http://bvsms.saude.gov.br/bvs/saudelegis/gm/20 13/prt0874_16_05_2013.html.

29. Matos JC, Pelloso SM, Carvalho MDB. Fatores associados à realização da prevenção secundária do câncer de mama no Município de Maringá, Paraná, Brasil. Cad Saúde Pública. 2011; 27(5):88898.

30. Murata IMH, Gabrielloni MC, Schirmer J. Cobertura do papanicolaou em mulheres de 25 a 59 anos de Maringá - PR, Brasil. Rev Bras Cancerol. 2012; 58:409-15.

31. World Health Organization. Early detection. Geneva: World Health Organization; 2007. (Cancer control : knowledge into action : WHO Guide for effective programmes ; module 3 ).

\section{CONTRIBUIÇÕES}

Joquebede Cristina Luchetti participou da concepção da pesquisa, coleta, análise dos dados e redação. Márcia Regina Campos Costa da Fonseca desenvolveu análise dos dados e revisão crítica. Maria Cristina Traldi orientou a pesquisa, delineamento, análise dos dados e, revisão crítica.

\footnotetext{
Como citar este artigo (Vancouver):

Luchetti JC, Fonseca MRCC, Traldi MC. Vulnerabilidade social e autocuidado relacionado à prevenção do câncer de mama e de colo uterino. REFACS [Online]. 2016 [citado em (inserir dia, mês e ano de acesso)]; 4(2). Disponível em: (link de acesso). DOI: 10.18554/refacs.v4i2.1639.

Como citar este artigo (ABNT):

LUCHETTI, J. C.; FONSECA, M. R. C. C.; TRALDI, M. C. Vulnerabilidade social e autocuidado relacionado à prevenção do câncer de mama e de colo uterino. REFACS, Uberaba, MG, v. 4, n. 2, p. 74-85, 2016. Disponível em: (link de acesso). DOI: 10.18554/refacs.v4i2.1639. Acesso em: (inserir dia, mês e ano de acesso).

Como citar este artigo (APA):

Luchetti, J. C., Fonseca, M. R. C. C. \& Traldi, M. C. (2016). Vulnerabilidade social e autocuidado relacionado à prevenção do câncer de mama e de colo uterino. REFACS, 4(2), 74-85. Recuperado em (dia), (mês), (ano) de (link de acesso). DOI: 10.18554/refacs.v4i2.1639.
} 